\title{
Existence and uniqueness of generalized monopoles in six-dimensional non-Abelian gauge theory
}

\author{
S.X.Chen ${ }^{\mathrm{a}, 1, *}$, L.Pei $^{\mathrm{b}}$ \\ ${ }^{a}$ School of Mathematics and Information Sciences, Henan University, Minglun Street, \\ Kaifeng, P.R.China 475001 \\ ${ }^{b}$ Arts and Science Experimental Class, Henan University, Minglun Street, Kaifeng, \\ P.R.China 475001
}

\begin{abstract}
In this paper, we established the existence and uniqueness of the spherically symmetric monopole solutions in $S O(5)$ gauge theory with Higgs scalar fields in the vector representation in six-dimensional Minkowski space-time and obtain sharp asymptotic estimates for the solutions. Our method is based on a dynamical shooting approach that depends on two shooting parameters which provides an effective framework for constructing the generalized monopoles in six-dimensional Minkowski space-time.
\end{abstract}

Keywords: generalized monopoles, dynamical shooting method, existence and uniqueness, six-dimension space-time, non-Abelian gauge theory 2000 MSC: 81T13, 65L10

\section{Introduction}

Long time ago, Dirac showed that quantum mechanics admits a magnetic monopole of quantized magnetic charge despite the presence of a singular Dirac string [4, 27]. Much later, G. 't Hooft and Polyakov showed that such magnetic monopoles emerge as regular configurations in $S O(3)$ gauge theory with spontaneous symmetry breaking triggered by triplet Higgs scalar

\footnotetext{
${ }^{*}$ Corresponding author

Email addresses: chensx1982@gmail.com (S.X.Chen), peilongsf@gmail.com (L.Pei)

${ }^{1}$ The research of the first author was supported in part by the Natural Science Fund of Henan Education Office (2007110004) and (2008A110002).
} 
fields [7, 21, 13, 22]. Although a monopole has not been detected or produced experimentally as a single particle, the existence of such objects has far reaching consequences.In the early universe, monopoles might have beed copiously produced, which vanished due to various physical interaction such as Pair production caused by Coulomb interaction among monopoles and anti-monopoles [5] but have significantly affected the history of the universe since then. For example, monopoles magnetic monopoles flow dispute the dynamo action, leading to a slow dynamo action in the best hypothesis or a decay of the magnetic field[6]. Also, Monopoles played a very important role in the formation of galaxy formation[17]. Gt Hooft-Polyakov monopoles emerge in grand unified theory of electromagnetic, weak, and strong interactions as well.

It is important and promising to explore solitonic objects in higher dimensional space-time under the super-string scenario, and recent extensive study of domain walls in super-symmetric theories, for instance, may have a direct link to the brane world scenario [9, 10, 11]. The energy of 't Hooft-Polyakov monopoles is bound from below by a topological charge. Monopole solutions saturate such bound, thereby the stability of the solutions being guaranteed by topology[1]. This observation prompts a question that if there can be a monopole solution in higher dimensions. Kalb and Ramond introduced Abelian tensor gauge fields coupled to closed strings [15]. Nepomechie showed that a new type of monopole solutions appear in those Kalb-Ramond antisymmetric tensor gauge fields 18]. Their implications to the confinement 23, 19, 20] and to ten-dimensional Weyl invariant space-time [8] has been explored. Topological defects in six dimensional Minkowski space-time as generalization of Dirac's monopoles were also found[28]. Tchrakian has investigated monopoles in non-Abelian gauge theory in higher dimensions whose action involves polynomials of field strengths of high degrees[24, 25]. Furthermore, it has been known that magnetic monopoles appear in the matrix model in the gauge connections describing Berry's phases on fermi states. In particular, in the $U S p$ matrix model they are described by $S U(2)$-valued anti-self-dual connections[12, 2]. H.Kihara and his team presented regular monopole configurations with saturated Bogomolny bound in $S O(5)$ gauge theory in six dimensions [16]. Self-gravitating Yang monopoles in all dimensions was also studied by G.W.Gibbons and P.K.Townsend[13].The purpose of this paper is to establish an existence and uniqueness theorem for these generalized monopoles in six-dimensional non-Abelian gauge.

In the next section, we first briefly discussed the mathematical structure 
of the problem of the existence of generalized monopoles in six-dimension non-Abelian gauge. We then state our main existence and uniqueness theorem for these solutions. In the third section, we transform the first-order equations into a second-order non-linear equation, and then we introduce a series of variable transformations to reduce the equation into a linear equation. In this case, the existence of generalized monopole solutions is seen to be equivalent to the existence problem of a nonlinear two-point boundary value problem. In section 4 , we present a dynamical shooting method which solves the existence problem completely and may be used as a constructive method for numerical computation. We shall also obtain sharp asymptotic estimates for the solutions.

\section{Mathematical Structure and Theorem}

Following Kihara, Hosotani and Nitta, we recall that a key to find correct Bogomolny equations in six-dimensional space-time is facilitated with the use of the Dirac or Clifford algebra. Let's consider $S O(5)$ gauge theory in sixdimensional space-time. Based on Clifford algebra and gauge transformation, the action is given by

$$
\begin{aligned}
I & =\int\left[-\frac{1}{8 \cdot 4 !} \operatorname{Tr} F^{2} * F^{2}-\frac{1}{8} \operatorname{Tr} D_{A} \phi-\frac{\lambda}{4 !}\left(\phi^{a} \phi_{a}-H_{0}^{2}\right)^{2} d^{6} x\right] \\
& =\int d^{6} x\left[-\frac{1}{8 \cdot 4 !} \operatorname{Tr}\left(F^{2}\right)_{\mu \nu \rho \sigma}\left(F^{2}\right)^{\mu \nu \rho \sigma}-\frac{1}{2} D_{\mu} \phi^{a} D^{\mu} \phi_{a}+\lambda\left(\phi^{a} \phi_{a}-H_{0}^{2}\right)^{2}\right],
\end{aligned}
$$

where the components of $F^{2}=\frac{1}{8}\left\{F_{\mu \nu}, F_{\rho \sigma}\right\} d x^{\mu} \wedge d x^{\nu} \wedge d x^{\rho} \wedge d x^{\sigma}$ are given by

$$
\begin{aligned}
& \left(F^{2}\right)_{\mu \nu \rho \sigma}=T_{\mu \nu \rho \sigma}^{e} \gamma_{e}-S_{\mu \nu \rho \sigma}, \\
& T_{\mu \nu \rho \sigma}^{e}(A)=\frac{1}{2 \cdot 4 !}{ }^{a b c d e}\left(F_{\mu \nu}^{a b} F_{\rho \sigma}^{c d}+F_{\mu \rho}^{a b} F_{\sigma \nu}^{c d}+F_{\mu \sigma}^{a b} F_{\nu \rho}^{c d}\right), \\
& S_{\mu \nu \rho \sigma}(A)=\frac{1}{4 !}\left(F_{\mu \nu}^{a b} F_{\rho \sigma}^{c d}+F_{\mu \rho}^{a b} F_{\sigma \nu}^{c d}+F_{\mu \sigma}^{a b} F_{\nu \rho}^{c d}\right) .
\end{aligned}
$$

The canonical conjugate momentum fields are given by

$$
\begin{aligned}
\Pi_{i}^{a b} & =\frac{\delta I}{\delta A_{i}^{a b}} \\
& =\frac{1}{3 !} T_{0 j k l}^{e} \frac{\delta T_{0 j k l}^{e}}{\delta F_{0 i}^{a b}}+\frac{4}{3 !} S_{0 j k} \frac{\delta S_{0 j k l}^{e}}{\delta F_{0 i}^{a b}} \\
& =\frac{1}{3}\left(M_{i, j k l}^{a b, e} M_{m, j k l}^{c d, e}+N_{i, j k l}^{a b, e} N_{m, j k l}^{c d, e}\right) F_{0 m}^{c d} \\
& :=U_{i, m}^{a b, c d} F_{0 m}^{c d},
\end{aligned}
$$

where $U$ is a symmetric, positive-definite matrix. To confirm the positivity of the Hamiltonian, we take the $A_{0}=0$ gauge in which $F_{0 i}^{a b}=A_{i}^{a b}$. It 
immediately follows that

$$
E=\int d^{5} x\left[\frac{1}{2} \Pi U^{-1} \Pi+\frac{1}{2 \cdot 4 !}\left(T_{i j k l}^{e}\right)^{2}+\left(S_{i j k l}\right)^{2}+H_{\phi}\right] \geq 0,
$$

where $H_{\phi}$ is the scalar field part of the Hamiltonian density.

In the $A_{0}=0$ gauge, the energy becomes lowest for static configurations $A_{i}^{a b}=\phi_{a}=0$ and it is given by

$$
\begin{aligned}
E & =\int d^{5} x \frac{1}{4 !}\left[\frac{1}{2}\left(T_{i j k l}^{e} \mp \epsilon^{i j k l m} D_{m} \phi^{e}\right)^{2}+\frac{1}{2}\left(T_{i j k l}^{e}\right)^{2}\right. \\
& \left. \pm \epsilon^{i j k l m} T_{i j k l}^{e} D_{m} \phi_{e}+\lambda\left(\phi_{a} \phi^{a}-H_{0}^{2}\right)^{2}\right] \\
& \geq \pm \int d^{5} x \frac{1}{4 !} \epsilon^{i j k l m} T_{i j k l}^{e} D_{m} \phi^{e} \\
& = \pm \int \operatorname{Tr} D_{A} \phi F^{2} \\
& :=\frac{16 \pi^{2}}{g^{2}} H_{0} \Psi .
\end{aligned}
$$

As $D_{A} F=0$ and thereby $\operatorname{Tr} D_{A} \phi F^{2}=d\left(\operatorname{Tr} \phi F^{2}\right), \Psi$ can be expressed as a surface integral

$$
\Psi= \pm \frac{g^{2}}{16 \pi^{2}} \int_{S^{4}} \operatorname{Tr} \phi F_{2}
$$

where $S^{4}$ is a space infinity of $R^{5}$.

The Bogomolny bound equation is

$$
*_{5}(F \wedge F)= \pm D_{A} \phi
$$

where $*_{5}$ is Hodge dual in five-dimensional space. In components, it is given by

$$
\begin{aligned}
\epsilon_{i j k l m} T_{i j k l}^{e} & = \pm D_{m} \phi_{e}, \\
S_{i j k l} & =0 .
\end{aligned}
$$

Let us define $e:=x^{a} \gamma_{a} / r$ and make a hedgehog ansatz[21]

$$
\begin{aligned}
& \phi=H_{0} U(r) e, \\
& A=\frac{1-k(r)}{2 g} e d e .
\end{aligned}
$$

It follows immediately that

$$
\begin{aligned}
D_{A} \phi & =H_{0}\left(K U d e+U^{\prime} e d r\right) \\
F & =\frac{1-K^{2}}{4 g} d e \wedge d e-\frac{K^{\prime}}{2 g} e d r \wedge d e .
\end{aligned}
$$

Accordingly, the boundary condition is

$$
U(\infty)= \pm 1, U(0)=0, K(\infty)=0, K(0)=1 .
$$


Applying $*_{5}(d e \wedge d e \wedge d e \wedge d e)=\frac{4 ! e d r}{r^{4}}$ and $*_{5}(e d r \wedge d r \wedge d r \wedge d r)=\frac{3 ! e d r}{r^{4}}$, the Bogomolny boundary equation(2.7)(with a plus sign)becomes

$$
\begin{aligned}
& K U=-\frac{\left(1-K^{2}\right) d K}{\tau^{2} d \tau}, \\
& \frac{d U}{d \tau}=\frac{\left(1-K^{2}\right)^{2}}{\tau^{4}}, \\
& U(\infty)=1, U(0)=0, K(\infty)=0, K(0)=1,
\end{aligned}
$$

where $\tau=a r, a=\left(\frac{2 g^{2}}{3} H_{0}\right)^{\frac{1}{3}}$.

In this case, $U$ increases as $\tau$ so that $U(\infty)=1$. A solution in the case $-D_{A} \phi=*_{5}(F \wedge F)$ is obtained by replacing $U$ by $-U$.

Our main existence and uniqueness theorem for generalized monopole solutions in the six-dimension non-Abelian gauge theory can be stated as follows:

Theorem 1. For any real number $g>0$ and $H_{0}>0$, the two point boundary value problem (2.12) has a unique solution $(K(r), U(r))$ so that $K(r)$ is strictly decreasing and $U(r)$ is strictly increasing for any $r>0$. Besides, there hold the sharp asymptotic estimates

$$
\begin{aligned}
& K=O\left(e^{-C r^{3}}\right), U=1+O\left(r^{-3}\right), r \rightarrow \infty, C>0 \\
& K=1+O\left(r^{2}\right), U=O(r), r \rightarrow 0 .
\end{aligned}
$$

This solution uniquely gives rise to a spherically symmetric finite-energy monopole solution of unit topological charge for non-Abelian gauge theory in six-dimensional Minkowski space-time.

\section{Second-Order Governing Equation}

Noting that the two equations in (2.12) can be combined to yield

$$
\frac{d\left(\frac{1-K^{2}}{\tau^{2} K}\right)}{d \tau} \frac{d K}{d \tau}+\frac{1-K^{2}}{\tau^{4}}=0
$$

or equivalently, in terms of $s=\ln \tau$ and $f(s)=K^{2}$ :

$$
f^{\prime \prime}-\left\{3+\frac{f^{\prime}}{f(1-f)}\right\} f^{\prime}+2 f(1-f)=0 .
$$


Accordingly, the boundary condition becomes

$$
f(-\infty)=1, f(\infty)=0 .
$$

We will prove that $0<f(s)<1, \forall s \in(-\infty, \infty)$. Note that $f=0$ and $f=1$ are two equilibrium solutions of equation (3.2), thus the existence and uniqueness theorem for solutions of ordinary differential equation allow us to see that $0<f<1, \forall s \in(-\infty, \infty)$. To make it convenient for us to solve our problem, we apply the transformation: $G(s)=\ln f(s)$. Under this transformation, $f^{\prime}$ and $f^{\prime \prime}$ can be represented as follows:

$$
f^{\prime}=e^{G} G, \quad f^{\prime \prime}=e^{G}\left(G^{\prime}\right)^{2}+e^{G} G^{\prime \prime} .
$$

Inserting (3.4) into (3.2), we have

$$
G^{\prime \prime}+\left(G^{\prime}\right)^{2}-\left\{3+\frac{G^{\prime}}{1-e^{G}}\right\} G^{\prime}+2\left(1-e^{G}\right)=0 .
$$

Meanwhile, it is easy to see that $-\infty<G<0$, and the boundary condition naturally becomes as follows:

$$
G(-\infty)=0, \quad G(\infty)=-\infty .
$$

Furthermore, we can see that equation (3.5) can be simplified to

$$
\left(G-e^{G}\right)^{\prime}-3\left(G-e^{G}\right)+2\left(1-e^{G}\right)^{2}=0 .
$$

To further simplify our problem, we introduce the transformation $V=G-$ $e^{G}$. Since the function $V(G)=G-e^{G}$ is strictly increasing in the interval $-\infty<G<0$, it is invertible, and its inverse function $Q(V)$ (say) enjoys the same properties over the interval $(-\infty,-1)$. In terms of the variable $V$, the equation (3.7) and its associated boundary condition becomes

$$
\begin{aligned}
& V^{\prime \prime}-3 V^{\prime}=-2\left(1-e^{G}\right)^{2}, s \in(-\infty, \infty), \\
& V(-\infty)=-1, V(\infty)=-\infty
\end{aligned}
$$

The equation (3.8) seems more tractable than equation (3.1) except that the function $Q(V)$ is not defined for $V \geq-1$, which makes it inconvenient to conduct a discussion. In order to fix this problem, we will make a suitable extension of the function $\left(1-e^{Q(V)}\right)^{2}$ to $V \geq-1$. Note that

$$
\lim _{V \rightarrow-1}\left(1-e^{Q(V)}\right)^{2}=\lim _{G \rightarrow 0}\left(1-e^{G}\right)^{2}=0 .
$$


Moreover, for $V<-1$, we have

$$
\frac{d}{d V}\left(1-e^{Q(V)}\right)^{2}=-2 e^{Q(V)}
$$

which tends to -2 as $V \rightarrow-1$. Hence, we can modify (3.8) into the following form,

$$
V^{\prime \prime}-3 V^{\prime}=R(V):= \begin{cases}-2\left(1-e^{Q(V)}\right)^{2}, & V<-1 \\ 4(V+1), & V \geq-1\end{cases}
$$

We see that $R(V)$ is a differentiable function for all $V$. We will consider (3.11) subject to boundary condition in (3.8). Although (3.11) alters the original equation in(3.8) due to its modified right-hand side function, we shall obtain a solution $V(s)$ that says negative for all $s \in(-\infty, \infty)$. In this way, we recover a solution to the original boundary value problem (3.8) as expected. Hence, our boundary value problem consisting of (3.11) and boundary condition in (3.8) becomes

$$
V^{\prime \prime}-3 V^{\prime}=R(V), s \in(-\infty, \infty), V(-\infty)=-1, V(\infty)=-\infty,
$$

where and in the sequel, we still use the prime' to denote the differentiation with respect to the variable $s$ when there is no risk of confusion.

\section{Mathematical Analysis}

To solve the two-point boundary value problem (3.12), we use a dynamical shooting method. This method was once used to solve problems [3, 26] in the field of mathematical physics. When we do this, we need to consider the initial value problem

$$
V^{\prime \prime}-3 V^{\prime}=R(V), s \in(-\infty, \infty), V(0)=m, V^{\prime}(0)=-n .
$$

Since we are looking for a solution $V<-1$, we naturally assume

$$
m<-1
$$

Under the assumption (4.2), we shall show that when $n$ is suitably chosen in (4.1), we may obtain a solution to (3.12). It can be seen from the structure of the problem that the boundary condition $V(-\infty)=-1$ is a crucial part 
to realize. So we shall look at this end first. For this purpose, we set $t=-s$ in the half interval $-\infty<s \leq 0$ and convert (4.1) into the form

$$
V^{\prime \prime}+3 V^{\prime}=R(V), t>0, V(0)=m, V^{\prime}(0)=n,
$$

where the prime' denotes the differentiation with respect to the reversed variable $t$. We also use $V_{t}$ to denote $\frac{d V}{d t}$. For fixed $m$ satisfying (4.2), we use $V(t ; n)$ to denote the unique solution of (4.3) which is defined in its interval of existence.

We are now ready to launch a shooting analysis for (4.3). We express the set of real numbers $R$ as the disjoint union of three data sets as follows:

$\beta^{-}=\left\{n \in R \mid\right.$ there exists $t>0$ so that $\left.V_{t}(t ; n)<0\right\}$,

$\beta^{0}=\left\{n \in R \mid V_{t}(t ; n)>0\right.$ and $V(t ; n) \leq-1$ for all $\left.t>0\right\}$,

$\beta^{+}=\left\{n \in R \mid V_{t}(t ; n)>0\right.$ for all $t \geq 0$ and $V(t ; n)>-1$ for some $t>0$ \} .

Lemma 2. We have the disjoint union $R=\beta^{-} \cup \beta^{0} \cup \beta^{+}$.

Proof. If $n \notin \beta^{-}$, then $V_{t}(t ; n) \geq 0$ for all $t$. If there exists a point $t_{0}>0$ so that $V_{t}\left(t_{0} ; n\right)=0$, then $V\left(t_{0} ; n\right) \neq 0$ because $V(t ; n)=0$ is an equilibrium point of the differential equation in (4.3) which is not attainable in finite time. Using the information $V_{t}\left(t_{0} ; n\right)=0$ but $V\left(t_{0} ; n\right) \neq 0$ in (4.3), we see that either $V^{\prime \prime}>0$ or $V^{\prime \prime}<0$ at $t=t_{0}$. Hence, there is a $t>t_{0}$ or $t<t_{0}$ at which $V_{t}(t ; n)<0$. This contradicts the assumption that $n \notin \beta^{-}$. Thus $V_{t}(t ; n)>0$ for all $t>0$ and $n \in \beta^{0} \cup \beta^{+}$, which proves the relation $R=\beta^{-} \cup \beta^{0} \cup \beta^{+}$as claimed.

Lemma 3. The set $\beta^{+}$and $\beta^{-}$are both open and nonempty.

Proof. The fact that $\beta^{-} \neq \emptyset$ follows immediately from the fact that $(-\infty, 0) \subset$ $\beta^{-}$. To see that $\beta^{+}$is nonempty, we integrate (4.3) to get

$$
\begin{gathered}
V_{t}(t ; n)=\left(n+\int_{0}^{t} R\left(V\left(s_{1} ; n\right) e^{3 s_{1}} d s_{1}\right) e^{-3 t},\right. \\
V(t ; n)=m+n\left(1-e^{-3 t}\right)+\int_{0}^{t} e^{-3 s_{1}}\left(\int_{0}^{s_{1}} R\left(V\left(s_{2} ; n\right)\right) e^{3 s_{2}} d s_{2}\right) d s_{1} .
\end{gathered}
$$

For any fixed $t_{0}>0$, we can choose $n>0$ sufficiently large so that

$$
V_{t}\left(t_{0} ; n\right)>0
$$




$$
V\left(t_{0} ; n\right)>-1
$$

Considering $V_{0}(0 ; n)=n>0, V(0 ; n)=m<-1$ and the property of continuous function, we can see that there exist a set of intervals $\left\{\left(0, \delta_{n}\right)\right\}$ so that $\left(0, \delta_{n}\right) \subset\left(0, \delta_{n+1}\right)$ and $V_{t}(t ; n)>0$ for all $t \in\left(0, \delta_{n}\right)$ where $n \in Z^{+}$. As the basis for the proof of this lemma, we will first prove that there exists $k \in Z_{+}$so that if we denote $t_{1}=\min \left\{\delta_{k}, t_{0}\right\}$, there holds $V_{t}(t ; n)>0$ for all $t \in\left(0, t_{1}\right]$ and $V(t ; n)<-1$ for all $t \in\left(0, t_{1}\right)$ but $V\left(t_{1} ; n\right)=-1$. Suppose otherwise that there exists no such $t_{1}$ satisfying the condition mentioned closely above. Denote $T=\sup \left\{\delta_{n}\right\}$ and it is easy to see that $V_{t}(T ; n)=0$, thereby $T \neq t_{0}$ because $V_{t}\left(t_{0} ; n\right)=0$. Therefore, we can divide the proof of the lemma into two sections according to whether $T<t_{0}$ or $T>t_{0}$.

First, if $T<t_{0}$, then the supposition mentioned closely above leads to $V(T ; n)<-1$ and $V_{t}(T ; n)=0$. Moreover, from the property of continuous function, we know that there exists $T_{0}>0$ so that $V(t ; n)<-1, \forall t \in(T, T+$ $T_{0}$ ). Therefore, it is easy to conclude that

$$
\begin{gathered}
V_{t}\left(T+\frac{T_{0}}{2}\right)<0, \\
V\left(T+\frac{T_{0}}{2}\right)<-1 .
\end{gathered}
$$

Clearly, the two inequities listed above together with the structure of $V_{t}(t ; n)$ and $V(t ; n)$ listed above allow us to see that for any $t \in\left(T+\frac{T_{0}}{2}, t_{0}\right)$ there hold

$$
\begin{gathered}
V_{t}\left(T+\frac{T_{0}}{2}\right)<0, \\
V\left(T+\frac{T_{0}}{2}\right)<-1 .
\end{gathered}
$$

which contradict (4.6) and (4.7), thus the lemma is proved provided that $T<t_{0}$.

Second, if $T>t_{0}$, the supposition mention above allows us to see that for any $t \in\left[0, t_{0}\right]$ there holds

$$
V(t ; n)<-1
$$

which also contradicts (4.7).

Considering the two cases, we can see that there exists $\delta_{k} \in\left(0, t_{0}\right)$ (We denote this $\delta_{k}$ as $\left.t_{1}\right)$ so that $V_{t}(t ; n)>0$ for any $t \in\left[0, t_{1}\right]$ and $V(t ; n)<-1$, $\forall t \in\left[0, t_{1}\right)$ but $V\left(t_{1} ; n\right)=-1$. 
We will then prove that $V_{t}(t ; n)>0$ for all $t \in[0, \infty)$. In fact, suppose otherwise that there exists $t_{3}>t_{1}$ so that $V_{t}(t ; n)>0$ for any $t \in\left[t_{1}, t_{3}\right)$ but $V_{t}\left(t_{3} ; n\right)=0$. Noting that $V(t ; n)>-1, \forall t \in\left[t_{1}, t_{3}\right]$ and considering (4.4), we can see that there holds

$$
n+\int_{0}^{t_{3}} R(V(t ; b)) e^{3 t} d t>n+\int_{0}^{t_{1}} R(V(t ; b)) e^{3 t} d t>0 .
$$

Therefore,

$$
\left(n+\int_{0}^{t_{3}} R(V(\tau ; b)) e^{3 \tau} d \tau\right) e^{-t_{3}}>0,
$$

which contradicts the fact that $V_{t}\left(t_{3} ; n\right)=0$. Hence, we know that $V(t ; n)>$ $0, \forall t \in[0, \infty)$. Consequently,we can conclude that $V(t ; n)>-1$ for all $t \in\left(t_{1}, \infty\right)$, and naturally, we can see that $V_{t}(t ; n)>0$ for all $t \in\left(t_{1}, \infty\right)$. Therefore, $n \in \beta^{+}$and the nonemptyness of $\beta^{+}$is established.

Moreover, for $n_{0} \in \beta^{+}$, there is a $t_{0}>0$ so that $V\left(t_{0} ; n_{0}\right)>-1$. By the continous dependence of $V$ on the parameter $n$ we see that when $n_{1}$ is close to $n_{0}$ we have $V_{t}\left(t ; n_{1}\right)>0$ for all $t \in\left[0, t_{0}\right]$ and $V\left(t_{0}, n_{1}\right)>-1$. As proved above, $V_{t}(t ; n)>0$ for all $t>t_{1}$. Thus, we can see that $V_{t}\left(t ; n_{1}\right)>0$ for all $t>t_{0}$ as well, which proves $n_{1} \in \beta^{+}$. So $\beta^{+}$is open. The fact that $\beta^{-}$is open is self-evident. The lemma follows.

Lemma 4. The set $\beta^{0}$ is a nonempty closed set. Moreover, if $n \in \beta^{0}$, then $V(t ; n)<-1$ for all $t>0$.

Proof. The first part of the lemma follows from the connectedness of $R$ and Lemma 4.2. To prove the second part, we assume otherwise that there is a $t_{0}>0$ so that $V\left(t_{0} ; n\right)=0$. Since $V(t ; n) \leq-1$ for all $t>0, V$ attains its local maximum at $t_{0}$. In particular, $V_{t}\left(t_{0} ; n\right)=0$, which contradicts the definition of $\beta^{0}$.

Lemma 5. For $n \in \beta^{0}$, we have $V(t ; n) \rightarrow-1$ as $t \rightarrow \infty$.

Proof. Since $V$ increases and $V<-1$ for all $t>0$, we see that the limit $\lim _{t \rightarrow \infty} V(t ; n)=V_{\infty}$ exists and $-\infty<V_{\infty} \leq 0$. If $V_{\infty}<0$, then $R(V(t ; n))<$ $R\left(V_{\infty}\right)<0$. Inserting this result into (4.4), we see that $V_{t}(t ; n)<0$ when $t>0$ is sufficiently large, which contradicts the definition of $\beta^{0}$.

Lemma 6. The set $\beta^{0}$ is actually a single point set. In other words, the correct shooting data is in fact unique. 
Proof. Suppose otherwise that there are two points $n_{1}$ and $n_{2}$. Let $V\left(t ; n_{1}\right)$ and $V\left(t ; n_{2}\right)$ be the corresponding solutions of (4.3). Then the function $w(t)=V\left(t ; n_{1}\right)-V\left(t ; n_{2}\right)$ satisfies the boundary condition $w(0)=w(\infty)=0$ and the equation

$$
w^{\prime \prime}(t)+w^{\prime}(t)=R^{\prime}(\xi(t)) w(t), 0<t<\infty,
$$

where $\xi(t)$ lies between $V\left(t ; n_{1}\right)$ and $V\left(t ; n_{2}\right)$ and $R^{\prime}(V)=\frac{d R(V)}{d V}>0(\forall V)$ in view of (3.10) and (3.11). Applying the maximum principle to (4.15), we conclude that $w(t) \equiv 0$, which contradicts the assumption that $n_{1} \neq n_{2}$.

For $n \in \beta^{0}$, we now consider the decay rate of $V(t ; n)$ as $t \rightarrow \infty$. To simplify our problem, we introduce the following transformation $v=V+1$. From the properties of the function $R(V)$, we see that the linearized equation of the differential equation in (4.3) around $v=0$ is $\theta^{\prime \prime}+3 \theta^{\prime}-4 \theta=0$, whose characteristic equation has the roots $\lambda=-4$ and $\lambda=1$. Hence, we see that for any $\epsilon \in(0,1)$, there is a constant $C(\epsilon)$ such that

$$
-C(\epsilon) e^{-4(1-\epsilon) t}<v(t ; n)<0, \forall t \geq 0 .
$$

Note that, modulo the positive small constant $\epsilon$, the above estimate is sharp. We now go back to the variable $s=-t$. Thus, we have obtained a solution $V(s)$ of (3.12) defined in the left of the real line, $-\infty<s \leq 0$, such that $V(s) \leq-1$ for all $s \leq 0$, and

$$
-1-C(\epsilon) e^{4(1-\epsilon) s}<V(s)<-1, \quad \forall s \leq 0 .
$$

We now consider the right half of the real line, $0 \leq s<\infty$. When $s$ is near zero, there hold $V^{\prime}(s)<0$ and $V(s)<-1$. Inserting these into (4.1) and using (3.11), we see that $V^{\prime \prime}(s)<0$ there. This property implies that the structure of the differential equation in (3.11) allows us to preserve the negative sign for all $V(s), V^{\prime}(s)$ and $V^{\prime \prime}(s)$. In particular, the solution $V(s)$ exists for all $s>0$ and $V(s)$ is strictly decreasing everywhere. From the structure of the function $R(V)$ on the right-hand side of the differential equation, we easily deduce that $V(\infty)=-\infty$. Hence, a solution of (3.12) is obtained. We now strengthen our conclusion by deriving the accurate blow-up rate for $V(s)$ as $s \rightarrow \infty$.

Integrating the differential equation in (4.1), we obtain

$$
e^{-3 s} V^{\prime}(s)=-n-2 \int_{0}^{s}\left(1-e^{Q(V)}\right)^{2} e^{-3 s_{1}} d s_{1} .
$$


From (3.10) we can see that the integral on the right-hand side of (4.18) is convergent for $s \rightarrow \infty$. Thus, we have the sharp expression

$$
V^{\prime}(s)=-(n+\sigma(s)) e^{3 s},
$$

where $\sigma(s)=2 \int_{0}^{s}\left(1-e^{Q(V)}\right)^{2} e^{-3 s_{1}} d s_{1}$ is a bounded increasing function in $[0, \infty)$ and $\sigma(0)=0$. Consequently, we find that $V(s)$ has the following asymptotic behavior

$$
V(s)=-(n+\sigma(s)) e^{3 s}, s \geq 0 .
$$

In other words, the function $V(s)$ blows up to $-\infty$ as fast as the function $-e^{3 s}$ as $s \rightarrow \infty$.

We need also get the asymptotic behavior of $V^{\prime}(s)$ as $s \rightarrow-\infty$. For this purpose, consider the representation (4.3) in terms of the variable $t=-s$. Using the estimate (4.16) and (3.9), we see that the factor in front of $e^{-3 t}$ on the right-hand side of (4.4) is bounded. This establishes $V_{t}=O\left(e^{-3 t}\right)$ as $t \rightarrow \infty$. Therefore, we obtain the asymptotic estimate

$$
V^{\prime}(s)=O\left(e^{3 s}\right), s \rightarrow-\infty .
$$

It is clear that Lemma 5 implies some kind of uniqueness property for the boundary value problem (3.12). More precisely, we state

Lemma 7. Up to translations, $s \mapsto s+s_{0}$, the two-point boundary value problem (3.12) has a unique solution.

Proof. Let $V_{1}$ and $V_{2}$ be two solutions of (3.12). Then they are all negativevalued and strictly decreasing and their behavior indicates that there exists a unique point $s_{0}$ so that $V_{1}(0)=V_{2}\left(s_{0}\right)$. Set $V_{3}(s)=V_{2}\left(s+s_{0}\right)$, then both $V_{1}$ and $V_{3}$ are solutions of the differential equation in $(3.12)$ and $V_{1}(0)=V_{3}(0)$. Using lemma 4.5, we have $V_{1}^{\prime}(0)=V_{3}^{\prime}(0)$. Applying the uniqueness theorem for the initial value problem of an ordinary differential equation, we have $V_{1} \equiv V_{3}$, namely, $V_{1}(s)=V_{2}\left(s+s_{0}\right)$ for all $s$ and the lemma follows.

Now consider the boundary behavior of the function $G=Q(V)$. Using $\frac{d G}{d V}=Q^{\prime}(V)=\frac{1}{\left(1-e^{G(V)}\right)}, G \rightarrow-\infty$ as $V \rightarrow-\infty$ and the L'Hospital rule, we have

$$
\lim _{s \rightarrow \infty} \frac{G(s)}{V(s)}=\lim _{s \rightarrow \infty} \frac{1}{\left(1-e^{Q(V)}\right)}=1 .
$$


Combining (4.20) and (4.22), we see that for any $\epsilon>0$ there is a number $S_{\epsilon}>0$ so that

$$
(1+\epsilon) V(s) \leq G(s) \leq(1-\epsilon) V(s), s \geq S_{\epsilon} .
$$

With this estimate, we can consider $G^{\prime}(s)$ in terms of $V^{\prime}(s)$ when $s \rightarrow \infty$. Indeed, using the relation between $G(s)$ and $V(s)$, we have, for sufficiently large $s>0$,

$$
G^{\prime}(s)=\left(1-e^{G(s)}\right)^{-1} V^{\prime}(s)=\left(1+e^{G(s)}+O\left(e^{2 G(s)}\right)\right) V^{\prime}(s) .
$$

Similarly, we need to consider the asymptotics of $G(s)$ and $G^{\prime}(s)$ as $s \rightarrow-\infty$. Using the relation $V=G-e^{G}$, we have

$$
V=-1-\frac{1}{2} G(s)^{2}+O\left(G(s)^{3}\right) .
$$

for $G(s)$ near zero. Applying (4.17) in (4.25), we obtain the estimate

$$
-C(\epsilon) e^{2(1-\epsilon) s}<G(s)<0
$$

where $\epsilon>0$ can be made arbitrarily small and $C(\epsilon)>0$ is a constant depending on $\epsilon$. Note again that, modulo $\epsilon$, the estimate (4.26) is sharp. In terms of $t=-s, G(t)=O\left(e^{-2(1-\epsilon) t}\right)$ as $t \rightarrow \infty$. Inserting this into (4.3) and noting that $R(V)=-2\left(1-e^{Q(V)}\right)^{2}=O\left(e^{-4(1-\epsilon) t}\right)$, we see that $V_{t t}+3 V_{t}=O\left(e^{-4(1-\epsilon) t}\right)$. From this we get the estimate $V(t)=O\left(e^{-4(1-\epsilon) t}\right)$. Note that (4.3) indicates that $V_{t t}>0$, since $V_{t}>0$. Hence $V_{t}$ is decreasing. Therefore, there holds

$$
V_{t}<V(t)-V(t-1)=O\left(e^{-4(1-\epsilon) t}\right), t \geq 1
$$

Consequently, returning to the variable $s=-t$, we obtain the improved estimate

$$
V^{\prime}(s)=O\left(e^{4(1-\epsilon) s}\right), \text { as } s \rightarrow-\infty,
$$

over (4.21). Inserting this result into the relation

$$
G^{\prime}(s)=\left(1-e^{G(s)}\right)^{-1} V^{\prime}(s)=-(1+O(G(s)))^{-1}(G(s))^{-1} V^{\prime}(s),
$$

we acquire the asymptotic estimate

$$
G^{\prime}(s)=O\left(e^{2(1-\epsilon) s}\right), \text { as } s \rightarrow-\infty
$$


which is compatible with (4.26).

We will then return to the original variable $r$ and give the asymptotic estimate of $U$ and $K$ in terms of $r$. Note that we once applied the variable transformation

$$
\tau=a r, s=\ln \tau,
$$

and the function transformation

$$
f(s)=K^{2}, G=\ln f, V=G-e^{G} .
$$

Hence, both $U$ and $K$ in the original boundary problem can be represented with $G$. Applying (4.23) and (4.24), and with the understanding that the arbitrarily small constant $\epsilon>0$ is omitted in the final expression to simplify the notation, we arrive at

$$
K=O\left(e^{-C r^{3}}\right), C>0, \text { as } r \rightarrow \infty .
$$

In fact, the second equation of (2.12) origins from

$$
\frac{d(U-1)}{d \tau}=\frac{\left(1-K^{2}\right)^{2}}{(\tau)^{4}}
$$

where $\tau=a r, a=\left(\frac{2 g^{2}}{3} H_{0}\right)_{\frac{1}{3}}>0$. Therefore, when $r \rightarrow \infty$, thereby $r \rightarrow \infty$, we can get

$$
\frac{d(U-1)}{d \tau}=O\left((\tau)^{-4}\right)
$$

It follows immediately that

$$
U=1+O\left((\tau)^{-3}\right)=1+O\left(r^{-3}\right) \text {, as } r \rightarrow \infty .
$$

Similarly, from (4.29) and (4.30) we can acquire

$$
K=1+O\left(r^{2}\right), U=O(r), \text { as } r \rightarrow 0 .
$$

The proof of Theorem 2.1 is now complete.

Lemma 8. The correct shooting slope, $-n<0$, depends on $m$ continuously and monotonically so that $n\left(m_{1}\right)>n\left(m_{2}\right)>0$ for $m_{1}<m_{2}<-1$. 
Proof. We have seen that for any given $m<-1$, there is a unique number $n>0$ so that the unique solution of the initial value problem (4.1) gives a negative valued solution $V$ which solves the two-point boundary value problem (3.12)(cf.lemma4.5). Thus we can denote this well-defined correspondence as $n=n(m)$ and $V=V_{m}$. We show that $n(m)$ is continuous with respect to $m<-1$. Let $\left\{m_{j}\right\}$ be a sequence in $(-\infty,-1)$ which converges to a number $m_{0}<0$. We need to prove that $n\left(m_{j}\right) \rightarrow n\left(m_{0}\right)$ as $j \rightarrow \infty$. Suppose otherwise that this is not true. Then, without loss of generality, we may assume that there is an $\epsilon_{0}>0$ so that $\left|n\left(m_{j}\right)-n\left(m_{0}\right)\right| \geq \epsilon$ for all $j=1,2, \ldots$. On the other hand, we can use lemma 4.6 to obtain a sequence $\left\{s_{j}\right\}$ so that $V_{m_{j}}(s)=V_{m_{0}}\left(s_{j}+s\right)$ for all $s$. In particular, $m_{j}=V_{m_{j}}(0)=V_{m_{0}}\left(s_{j}\right)$ for $j=1,2, \ldots$ It is clear that $\left\{s_{j}\right\}$ is a bounded sequence otherwise it would contradict the assumption $m_{j} \rightarrow m_{0}<0(j \rightarrow 0)$ and the fact that $V_{m_{0}}(-\infty)=-1$ and $V_{m_{0}}(\infty)=-\infty$. By extracting a subsequence if necessary, we may assume that $s_{j} \rightarrow$ some $s_{0}$ as $j \rightarrow \infty$. Therefore, we have, as $j \rightarrow \infty, n\left(m_{j}\right)=V_{m_{j}}^{\prime}(0)=V_{m_{0}}^{\prime}\left(s_{j}\right) \rightarrow V_{m_{0}}^{\prime}\left(s_{0}\right):=n_{0} \neq n\left(m_{0}\right)$. On the other hand, $m_{j} \rightarrow-1$ as $j \rightarrow \infty$, and $m_{j}=V_{m_{j}}(0)=V_{m_{0}}\left(s_{j}\right)$ for $j=1,2, \ldots$ imply that $s_{j} \rightarrow 0$ as $j \rightarrow \infty$ since $V_{m_{0}}$ is strictly monotone. Hence $s_{0}=0$ and we arrive at a contradiction.

The continuous dependence of $n(m)$ on $m$ implies that the solution $V_{m}$ depends on $m$ continuously as well.We claim that $n(m) \rightarrow 0$ as $m \rightarrow 0^{-}$. Otherwise there is a sequence $\left\{m_{j}\right\}$ in $(-\infty,-1)$ and an $\epsilon_{0}$ so that $m_{j} \rightarrow 0$ as $j \rightarrow \infty$ but $n\left(m_{j}\right) \geq \epsilon_{0}(j=1,2, \ldots)$. Using these in the initial value problem (4.3) with $m=m_{j}$ and $n=n\left(m_{j}\right)$, we observe that the solution will assume a positive value for a slightly positive $t$ when $j$ is sufficiently large, which contradicts the definition of $n\left(m_{j}\right)$.

We can also claim that $n(m) \rightarrow \infty$ as $m \rightarrow-\infty$. Let $V_{0}$ be a fixed solution of (3.12). Then there is a unique $s_{m}$ so that $V_{m}(s)=V_{0}\left(s_{m}+s\right)$ (cf. lemma6). Since $m=V_{m}(0)=V_{0}\left(s_{m}\right)$, we conclude that $s_{m} \rightarrow \infty$ as $m \rightarrow-\infty$. Consequently, $n(m)=-V_{m}^{\prime}(0)$ as $m \rightarrow-\infty$ as claimed.

Remarks Our analysis suggests a dynamical shooting method for constructing the unique solution of the generalized monopole problem in sixdimension non-Abelian gauge. We have seen that we may start from the initial value problem (4.1) with an arbitrary $m<0$. The sets of undesired shooting data, $\beta^{-}$and $\beta^{+}$are two open intervals $\beta^{-}=(-\infty, n)$ and $\beta^{+}=(b, \infty)$. The correct shooting slope, $-n<0$, depends on $m$ continuously and monotonically so that $n\left(m_{1}\right)>n\left(m_{2}\right)>0$ for $m_{1}<m_{2}<-1$. 


\section{Acknowledgments}

The research of the first author was supported in part by the Natural Science Fund of Henan Education Office (2007110004) and (2008A110002).

\section{References}

[1] E. B. Bogomolny, Stability of Classical Solutions, Yad. Fiz. 24, 861870 (1976), Sov. J. Nucl. Phys., 35 (1976), pp. 449-454.

[2] B. Chen, H. Itoyama And H. Kihara, NonAbelian monopoles from matrices: Seeds of the space-time structure, Nucl. Phys. B, 577 (2000),pp. 23-46.

[3] S. Chen, D. Zu AND M. CAI, Existence of finite-energy electroweak monopoles, J. Math. Anal. Appl.,358 (2009), pp. 10-17.

[4] P. A. M. Dirac, The Theory of Magnetic Poles, Phys.Rev., 74 (1948), pp. 817-830.

[5] V. K. Dubrovich And N. A. Sushko, Magnetic Monopoles in the Early Universe: Pair Production, Astrophysics, 46 (2004), pp. 422-428.

[6] De Andrade. Garcia, Slow dynamos and decay of monopole magnetic fields in the early Universe, arXiv:0901.1714.

[7] G. 'T Hooft, Magnetic monopoles in unified gauge theories, Nucl. Phys. B, 79 (1974), pp. 276-284.

[8] Y. Hosotani, Weyl Invariant Spacetime, Prog. Theoret. Phys., 109 (2003), pp. 295-303.

[9] Y. Isozumi, M. Nitta, K. Ohashi and N. Sakai, Construction of Non-Abelian Walls and their Complete Moduli Space, Phys. Rev. Lett., 93, (2004), 161601.

[10] Y.Isozumi, M.Nitta, K.Ohashi And N.SAkai, All exact solutions of a 1/4 Bogomolnyi-Prasad-Sommerfield equation, Phys. Rev. D, 71 (2005), 065018. 
[11] Y. Isozumi, M. Nitta, K. Ohashi And N. SAkai, Non-Abelian walls in supersymmetric gauge theories, Phys. Rev. D, 70 (2004), 125014.

[12] H. Itoyama and T. Matsuo, Berry's Connection and USp(2k) Matrix Model, Phys. Lett. B, 439 (1998), pp. 46-54;

[13] G.W.Gibbons and P.K.Townsend, Self-gravitating Yang monopoles in all dimensions Class. Quantum Grav., 23 (2006), pp. 4873-4885.

[14] B. Julia And A. Zee, Poles with both magnetic and electric charges in non-Abelian gauge theory, Phys. Rev. D, 11 (1975), pp. 2227-2232.

[15] M. Kalb And P.'RAmond, Classical direct interstring action, Phys.Rev. D9 (1974), pp. 2273-2284.

[16] H. Kihara, Y. Hosotani, and M. Nitta, Generalized monopoles in six-dimensional non-Abelian gauge theory, Phys. Rev. D, 71 (2005), 041701.

[17] J. Madsen, Galaxy formation in a monopole dominated universe, Phys. Lett. B, 143 (1984), pp. 363-367.

[18] R. I. Nepomechie, Magnetic monopoles from antisymmetric tensor gauge fields, Phys.Rev. D, 31 (1985), pp. 1921-1924.

[19] P. ORLand, Instantons and disorder in antisymmetric tensor gauge fields, Nucl.Phys. B, 205 (1982), pp. 107-118.

[20] R. B. Pearson, Partition function of the Ising model on the periodic 444 lattice, Phys.Rev. B, 26 (1982), pp. 6285-6290.

[21] A. M. Polyakov, Particle Spectrum in Quantum Field Theory, JETP Lett., 20 (1974), pp, 194-195.

[22] M. K. Prasad and C. M. Sommerfield, Exact Classical Solution for the 't Hooft Monopole and the Julia-Zee Dyon, Phys. Rev. Lett., 35 (1975), pp. 760-762.

[23] R. SAvit, Topological Excitations in U(1)-Invariant Theories, Phys.Rev.Lett., 39 (1977), pp. 55-58. 
[24] D. H.Tchrakian, $N$ dimensional instantons and monopoles, J.Math.Phys., 21 (1980), pp. 166-172.

[25] D. H. Tchrakian and F. Zimmerschied, 't Hooft tensors as KalbRamond fields of generalized monopoles in all odd dimensions: $d=3$ and $d=5$, Phys.Rev. D,62 (2000), 045002.

[26] X. Wang And Y. YAng, Existence of Static BPS monopoles and Dyons in Arbitary (4p-1)-Dimensional Spaces, Lett. Math. Phys., 77(2006), pp. 249-263 .

[27] T. T. Wu And C. N. YAng, Multiparticle Quantum Mechanics Obeying Fractional Statistics, Phys.Rev.D, 12 (1975), pp. 3845-3857.

[28] C. N. YAnG, Generalization of Dime's monopole to SU(2) gauge fields, J.Math.Phys., 19 (1978), pp. 320-328. 